\section{Difference in Patterns of Psychiatric Emergencies in Different Cultures: Do Psychia- tric Residents Need More Than Written in Books?}

Sir,

Psychiatric emergencies make a big portion of emergency presentations in all parts of the world. Epidemiology of usual medical and surgical emergencies remain similar across the Globe, but psychiatric presentations may differ in different socio-cultural backgrounds. ${ }^{1} \quad$ Psychiatric emergencies include mental and behavioural disorders due to substance use, suicidal ideations or attempts, self-harm, psychotic episodes, mood disorders, extra-pyramidal symptoms, acute anxiety symptoms and pseudo-seizures or conversion disorder. ${ }^{2}$ Working as a psychiatric resident doctor and registrar in big teaching hospitals of Pakistan and Ireland not only broadened the horizon regarding basic knowledge of mental health, but also enabled to understand the difference in symptomatology, presentation and management of mental health patients in context of two entirely different cultures. Acute stress reaction, acute anxiety symptoms, and conversion disorder may be the top most psychiatric diagnosis in which most psychiatric emergencies could be fitted in at a tertiary care hospital of Islamabad, while mental and behavioural disorder due to substance use and self-harm were most commonly encountered conditions in emergency department of a teaching hospital in Dublin city centre.

Difference in socio-cultural milieu deeply affects the cognitions and behaviours of individuals and patients suffering from mental health problems are no exception to this. Patients using multiple substances with clear social adversities come to emergency department (ED) with low mood and suicidal ideation. ${ }^{3}$ In a lot of cases, they mention categorically that they would feel safe if admitted in the ward, but would end their life if discharged from ED. Eliciting the requirement of crisis admission, presence of axis-1 diagnosis, evidence of recent substance use and chalking out the appropriate emergency care plan are usually main goals of most of ED psychiatric emergency assessments in the West. In Eastern world, it is usually stress reaction, acute anxiety and conversion disorder which has been encountered by resident psychiatrists as emergency consults. ${ }^{4}$ Carefully ruling out organic condition, identifying the recent stressor and addressing the concerns of family are the real challenges faced by the duty resident psychiatrist after the emergency assessments in our part of the world. For working in two different cultures with different kinds of emergency psychiatric presentations, stressors and social set-up, a different kind of training is required for the resident doctors to manage the patients; and sometimes, whole situation effectively.

An interesting local study published in 2017 highlighted the fact that psychogenic syncope, pseudo-seizures, aphonia, and paralysis or muscle weakness were common conversion features found in patients presenting to ED. ${ }^{5}$ Statistics from UK were different in this regard; where self-harm and mental and behavioural disorders due to substance use were most commonly encountered mental health presentation. ${ }^{3}$ Analysing the existing literature and incorporating own experience of working in ED of tertiary care hospitals of Eastern and Western countries, it is recommended that in addition to bookish psychiatric knowledge, the junior psychiatric doctor on call must be aware of local patterns of mental health presentations, cultural values and norms, support systems; and most importantly, hierarchy of decision-making for formulation of immediate, short term, and long term care plans.

\section{CONFLICT OF INTEREST:}

The author declared no conflict of interest.

\section{AUTHOR'S CONTRIBUTION:}

This letter is written solely by the author.

\section{REFERENCES}

1. Barratt H, Rojas-García A, Clarke K, Moore A, Whittington C, Stockton $S$, et al. Epidemiology of mental health attendances at emergency departments: Systematic review and meta-analysis. PLoS One 2016; 11(4):e0154449. doi:10.1371/journal.pone.0154449.

2. Adeosun II, Adegbohun AA, Jeje 00, Oyekunle 00, Omoniyi MO. Urgent and nonurgent presentations to a psychiatric emergency service in Nigeria: Pattern and correlates. Emerg Med Int 2014; 2014:479081. doi:10.1155/2014/ 479081.

3. Duncan EAS, Best C, Dougall N, Skar S, Evans J, Corfield AR, et al. Epidemiology of emergency ambulance service calls related to mental health problems and self harm: A national record linkage study. Scand J Trauma Resusc Emerg Med 2019; 27(1):34. doi: 10.1186/s13049-0190611-9.

4. Hanif R, Afridi MI, Dars JA, Iqbal F. Pattern of presentations at psychiatric emergency services of a public sector tertiary care hospital, Pakistan. J Pak Med Assoc 2020; 70(12(A):2132-8. doi: 10.47391/JPMA.08-805.

5. Amin R, Dar MM, Maqbool S, Paul MA, Kawoos Y, Zargar W. Clinical presentation and life events in patients with functional neurological symptom disorder presenting to psychiatric emergency OPD - A hospital based study. Int J Contemp Med Res 2017; 4(12):2393-9. 


\section{Usama Bin Zubair}

. D.............

Department of Liasion Psychiatry, Connolly Hospital, Dublin, Ireland

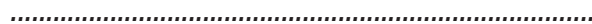

Correspondence to: Dr. Usama Bin Zubair, Department of
Liasion Psychiatry, Connolly Hospital, Dublin, Ireland E-mail: drusamabinzubair@yahoo.com

....................................................................

Received: February 10, 2021; Revised: March 12, 2021; Accepted: March 16, 2021

DOI: https://doi.org/10.29271/jcpsp.2022.02.268

\footnotetext{
$\bullet \bullet \bullet \bullet \bullet \bullet \bullet \bullet \bullet$
} 